\title{
Lagrangian tracers on a surface flow: the role of time correlations
}

\author{
Guido Boffetta ${ }^{1}$, Jahanshah Davoudi ${ }^{2}$, Bruno Eckhardt ${ }^{2}$ and Jörg Schumacher ${ }^{2}$ \\ ${ }^{1}$ Dipartimento di Fisica Generale and INFM, Università di Torino, Via Pietro Giuria 1, I-10125 Torino, Italy and \\ 2 Fachbereich Physik, Philipps-Universität, Renthof 6, D-35032 Marburg, Germany
}

\begin{abstract}
Finite time correlations of the velocity in a surface flow are found to be important for the formation of clusters of Lagrangian tracers. The degree of clustering characterized by the Lyapunov spectrum of the flow is numerically shown to be in qualitative agreement with the predictions for the whitein-time compressible Kraichnan flow, but to deviate quantitatively. For intermediate values of compressibility the clustering is surprisingly weakened by time correlations.
\end{abstract}

PACS numbers: 47.27.-i, 47.27.Ak, 05.40.-a

Inhomogeneous distribution of particles advected in a turbulent flow is a generic consequence of compressibility. This can be obtained in two situations. The first possibility is that the advecting flow is compressible itself and that the particles follow the streamlines [1]. The other possibility is that the particles do not follow the streamlines because of inertia $[2,3,4,5,6,7,8]$ or lift [9], and that the effective velocity field is compressible. Such situations are relevant for the formation of clouds [10] or for the advection of bubbles in turbulent flows, e.g. for breaking waves on the ocean surface $[11,12]$. We focus here on the first possibility.

While the dominant tendency of incompressible flows is to separate particle trajectories, a compressible component is responsible for particle trapping in contracting regions for long times. The Eulerian compressibility of a flow is measured by the dimensionless ratio $\mathcal{C}=\left\langle\left(\partial_{i} u_{i}\right)^{2}\right\rangle /\left\langle\left(\partial_{i} u_{j}\right)^{2}\right\rangle$. It takes values between 0 (incompressible flow) and 1 (potential flow). While there can be no clustering without compressible effects, the compressibility ratio $\mathcal{C}$ is insufficient to determine the final distribution completely [13]: the behavior depends also on the spatial roughness, the dimensionality, and, as we will demonstrate here, on the time correlations in the flow.

A convenient characterization of the final distribution uses the Lagrangian Lyapunov spectrum. Dynamical systems theory shows that the asymptotic clusters are smooth along the unstable directions of positive Lyapunov exponents and fractal along the stable directions. In $d$ dimensions, the sum of Lyapunov exponents $\sum_{i=1}^{d} \lambda_{i}$ vanishes in the incompressible case and is negative for a compressible flow. A measure for the distribution of the final clusters is given by the Lyapunov dimension [14],

$$
D_{L}=K+\frac{\sum_{i=1}^{K} \lambda_{i}}{\left|\lambda_{K+1}\right|},
$$

where $\mathrm{K}$ is the maximal integer such that $\sum_{i=1}^{K} \lambda_{i} \geq 0$. Obviously, for an incompressible flow $K=d$ and also $D_{L}=d$ : the particle distribution fills the entire volume. As compressibility increases, the sum of Lyapunov exponents will become negative and $K$ will drop below $d$.
If the largest Lyapunov exponent $\lambda_{1}$ becomes negative, the final cluster will not have any smooth directions anymore and the particles will cluster in a point-like fractal. One hence distinguishes a regime of strong compressibility $\lambda_{1}<0$ with $D_{L}=0$, and one of weak compressibility $\lambda_{1}>0$.

There are no general results on the spectrum of Lagrangian Lyapunov exponents in turbulent flows. For the case of incompressible, isotropic, three dimensional turbulence the numerical observation is that $\lambda_{2} \sim$ $1 / 4 \lambda_{1}$ [15]. In the limit of a compressible Kraichnan flow, which is a synthetic, white-in-time, and Gaussian distributed, the spectrum is given by $\lambda_{j}=$ $C_{1}[d(d-2 j+1)-2 \mathcal{C}(d+(d-2) j)]$ where $j=1, \ldots, d$, $C_{1}$ is an inverse time proportional to the Lagrangian strain and the resulting Lyapunov dimension is a decreasing function of $\mathcal{C}$ [13]. Moreover, when $\mathcal{C} \geq \mathcal{C}_{s}=d / 4$ all the Lyapunov exponents become negative and one has the strong compressible regime.

The trapping effects are believed to be enhanced in spatially rough Kraichnan flows [13]. Although a recent attempt has been devoted to re-introduce the finite time correlation for the synthetic rough Kraichnan flows [16] a general theoretical framework studying the effects of the time correlation is still lacking.

Here, we demonstrate numerically the crucial role of finite time correlations in the Lagrangian statistics of particles transported by the Navier-Stokes flow which is established in a free-slip surface. As a consequence of boundary conditions the two-dimensional flow displays an effective compressibility. By decomposing the surface velocity field in its irrotational and potential components, we change the effective value of compressibility of the surface flow. This enables us to study the interplay of the two components, time correlations and compressibility, within one system.

We want to remark that our investigation does not encounter the effects of fluid density variations which appear in a compressible turbulent flow.

The tracers are advected by the free surface flow on top of three-dimensional $(3 d)$ incompressible Navier-Stokes turbulence at $R e_{\lambda} \simeq 145$. The volume with an aspect 


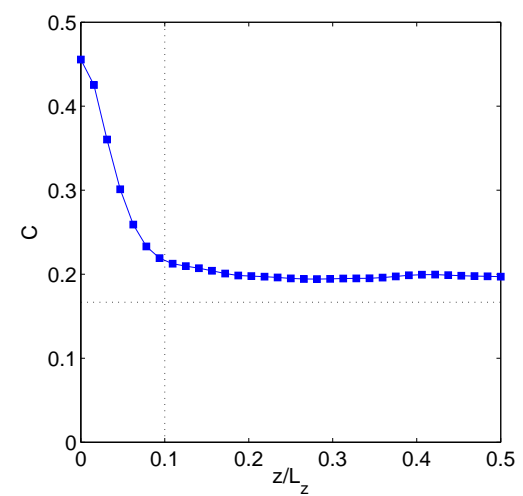

FIG. 1: Compressibility $\mathcal{C}$ as a function of $\operatorname{depth} z / L_{z}$ ranging from the free surface $\left(z / L_{z}=0\right)$ to the middle of the tank $\left(z / L_{z}=0.5\right)$. The figure illustrates also that for $z / L \geq 0.1$ the volume turbulence gets close to the homogeneous isotropic case which is indicated by the vertical dotted line. The horizontal dotted line indicates the $\mathcal{C}$ value of $1 / 6$ which would follow for perfect isotropy in the bulk.

ratio of $2 \pi: 2 \pi: 1$ is resolved by $256 \times 256 \times 65$ grid points. The full $3 d$ equations are integrated by a standard pseudo-spectral method [17]. The lateral boundary conditions for $x$ and $y$ are periodic and in the vertical direction we apply free-slip boundary conditions, $u_{z}=0$ and $\partial_{z} u_{x}=\partial_{z} u_{y}=0$. The Lagrangian tracers are advected by the surface flow spanned by the two components $u_{x}(x, y, z=0, t)$ and $u_{y}(x, y, z=0, t)$ which is compressible since $\partial_{x} u_{x}+\partial_{y} u_{y}=-\partial_{z} u_{z} \neq 0$ [17]. Tracer velocity between the grid mesh is calculated by bi-linear interpolation. We store 18,000 configurations of the $2 d$ surface velocity field at the same resolution in time that is used for the time advancement of the full $3 d$ Navier-Stokes equations. On the basis of these configurations, Lagrangian trajectories and the Lyapunov spectrum $\left(\lambda_{1}, \lambda_{2}\right)$ are computed.

Isotropy can be used to show that the compressibility ratio for a two-dimensional flow $\left(u_{x}(x, y), u_{y}(x, y)\right)$ can be rewritten as

$$
\mathcal{C}=\frac{\left\langle\left(\partial_{x} u_{x}+\partial_{y} u_{y}\right)^{2}\right\rangle}{2\left\langle\left(\partial_{x} u_{x}-\partial_{y} u_{y}\right)^{2}\right\rangle} .
$$

Numerical and experimental investigations of the surface flow at Taylor Reynolds numbers $R e_{\lambda} \sim 10^{2}$ have shown that $\mathcal{C} \simeq 0.5[18,19]$. By $(2)$, this is equivalent to a vanishing value of the cross correlation $\left\langle\partial_{x} u_{x} \partial_{y} u_{y}\right\rangle$, as confirmed in numerical studies [20]. Figure 1 shows the profile of $\mathcal{C}$ for all planes of the simulation box from $z=0$ down to $z=L / 2$. It is interesting to note that the value of compressibility measured in the bulk is very close to the value $1 / 6$ which is expected for a two-dimensional cut of a three-dimensional homogeneous, isotropic flow. The relatively large value of the compressibility ratio on the free surface is a consequence of the free-slip boundary conditions.
The evolution of an initially uniform particle distribution in the time-correlated surface flow with $\mathcal{C}=0.45$ is shown at two instants in Figs. $2 a$ and $2 c$, respectively. The pronounced formation of particle clusters was observed recently in experiments [18] and in simulations [19].
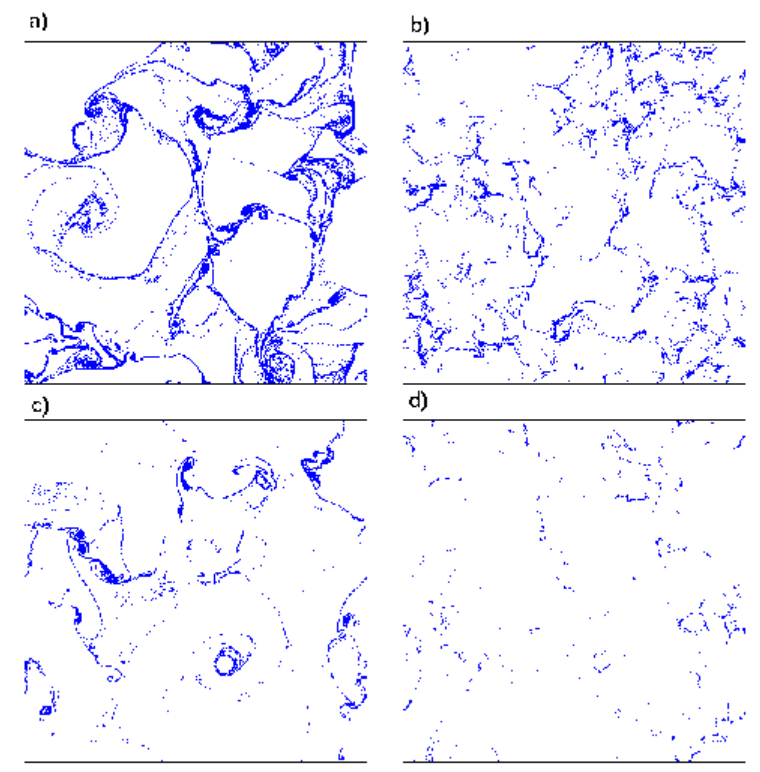

FIG. 2: Visualization of the particle clustering for a uniform initial distribution of 36,000 tracers on the free surface $z=$ 0 with $\mathcal{C}=0.45$. The panels $(a)$ and $(c)$ show the tracer distributions in time correlated surface flow at $0.2 T$ and $3.1 T$. The panels $(b)$ and $(d)$ correspond to the tracer distributions in the time de-correlated flow at $0.2 T$ and $5.1 T$. The time scale $T=L / \delta u(L)$ is the large scale eddy turnover time.

Although having a compressibility close to the critical value of $\mathcal{C}_{2}=1 / 2$ for the two-dimensional Kraichnan flow we do not observe point-like structures; particles accumulate on a network of narrow ridges with bigger empty voids in between. Figure 3 reports the results of the numerical computation of the Lagrangian Lyapunov spectrum. The very existence of the ridge structures pertains to the existence of a finite positive Lyapunov exponent $\lambda_{1} \sim 0.3$. This is in line with the computed Lyapunov dimension, $D_{L} \simeq 1.15$ which is plotted in the inset of Fig. 3.

We now compare the Lyapunov dimensions of the timecorrelated flow with the the corresponding de-correlated flow. That would quantify the effects of time correlation effects on clustering properties. In order to do so, we generate the decorrelated velocity field from the flow at hand by re-shuffling the temporal sequence of surface flow snapshots. Thanks to the periodic boundary conditions, each snapshot is further de-correlated by phase randomization in space.

Figures $2 b$ and $2 d$ show the evolution of the density of 


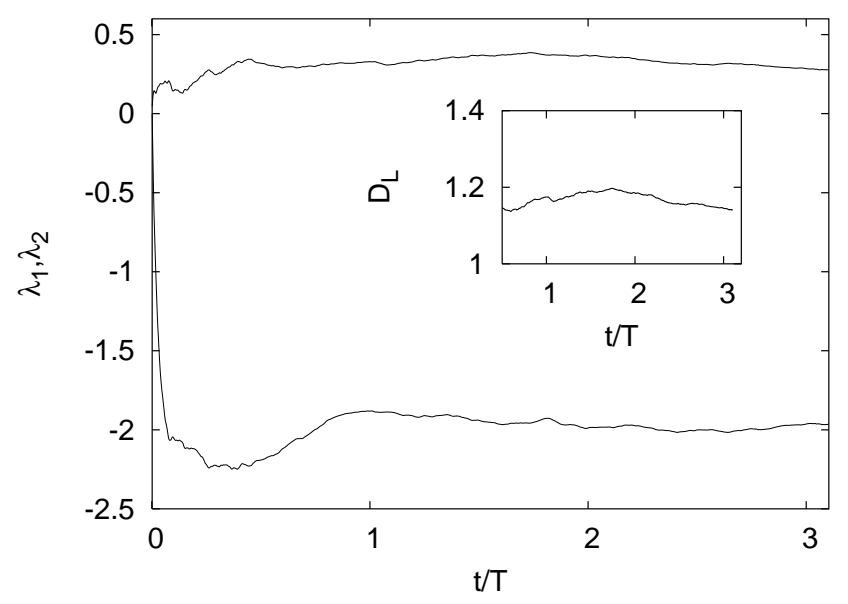

FIG. 3: Convergence of Lyapunov exponents $\left(\lambda_{1}>0\right.$ and $\left.\lambda_{2}<0\right)$ for the Lagrangian trajectories on the free surface computed for about three eddy turnover times $T$. In the inset, the Lyapunov dimension $D_{L}=1+\lambda_{1} /\left|\lambda_{2}\right|$ is shown.

tracers in the re-shuffled time de-correlated flow. We find the clusters concentrated on more point-like sets and the ridges are depleted evidently. Therefore one expects that the Lyapunov dimension is decreased when the temporal correlation of the velocity is diminished. Lagrangian trajectories in the randomized flow are more erratic: in order to achieve statistical convergence, trajectories are now integrated for about 5 large scale eddy turnover times. The computation of Lyapunov spectrum and dimension for the randomized flow is shown in Figure 4. The fact that both of the Lyapunov exponents are changed with respect to Fig. 3 is not relevant, as the characteristic Lagrangian time is changed by time decorrelation procedure. The important point is that the Lyapunov dimension is now $D_{L} \simeq 1.05$ which is less than the Lyapunov dimension in the time correlated flow. This is quite surprising as one intuitively expects that the particle trapping is enhanced when moving in a time-correlated flow because the local flow will persist for some time.

In the case of two-dimensional compressible Kraichnan ensemble the pairing symmetry in the Lyapunov spectrum $(\lambda,-\lambda)$ is broken and the Lyapunov dimension is then simply given by

$$
D_{L}=\frac{2}{1+2 \mathcal{C}}
$$

According to (3), the transition to the strong compressibility regime takes place at $\mathcal{C}_{s}=1 / 2$, very close to the numerically observed compressibility at the surface (see Fig. 1). The prediction of (3) for $\mathcal{C} \simeq 0.45$ renders $D_{L} \sim 1.06$ which is in very good agreement with the result of randomized surface flow. The compressibility, seen along Lagrangian trajectories [4], is different from $\mathcal{C}$ as given by (2). However explicit theoretical results inferring to the effects of velocity time correlations on

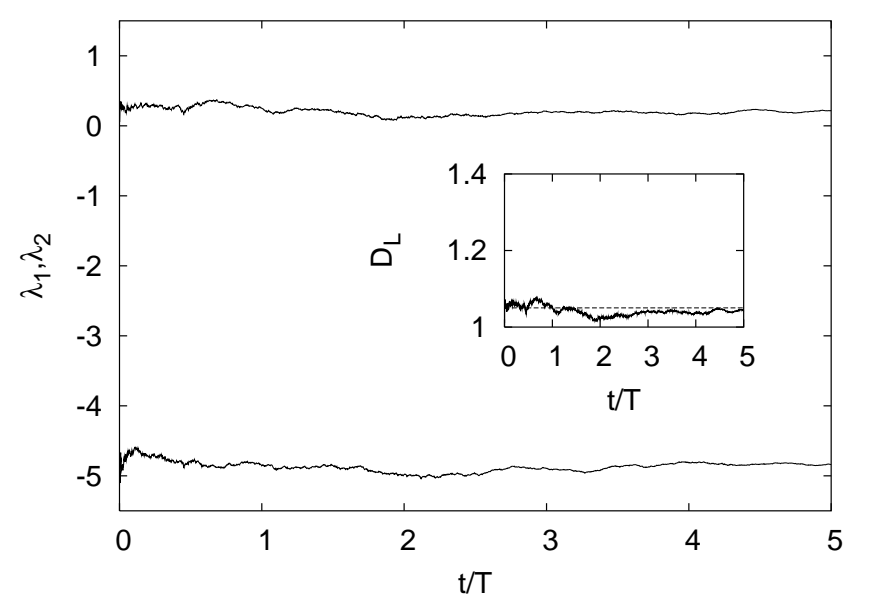

FIG. 4: Convergence of Lyapunov exponents for the Lagrangian trajectories advected by the random-time, randomphase scrambled velocity field. The computation is done for $5 T$. In the inset, we show the Lyapunov dimension $D_{L}$ together with the theoretical prediction $D_{L}=1.05$ (dashed line).

effective Lagrangian compressibility or Lyapunov spectrum are still lacking although first attempts have been made [16]. Moreover, it is not clear that the theoretical description of the time correlated flows will enjoy the same level of generality.

In order to gain more insight on the role of the time correlations we repeated the Lagrangian analysis for different degrees of compressibility. To this end, we have decomposed the $2 d$ velocity snapshots into the stream function $\psi(x, y)$ and the potential function $\phi(x, y)$. A new velocity field is reconstructed then to

$$
\tilde{\mathbf{u}}(x, y)=\sqrt{2}\left(\nabla^{T} \psi(x, y) \cos \gamma+\nabla \phi(x, y) \sin \gamma\right)
$$

where $\nabla^{T}=\left(\partial_{y},-\partial_{x}\right)$. The compressibility ratio $\mathcal{C}$ (cf. (2)) for the decomposed flows is a monotonic function of the parameter $\gamma \in[0, \pi / 2]$ and for $\gamma=\pi / 4$ the original surface flow is reproduced. $\gamma=0$ corresponds to the incompressible case and $\gamma=\pi / 2$ gives a purely potential flow; so we are able to cover the whole range of compressibility degrees. Of course, dimensional quantities such as the characteristic Lagrangian time of the velocity field may depend on the value of $\gamma$. On the other hand, dimensionless quantities, such as $D_{L}$ are independent on a global rescaling of the velocity and thus can be expected to depend mainly on the degree of compressibility.

We have performed two sets of numerical simulations of Lagrangian trajectories for different values of $\gamma$. For each value, we have computed the mean compressibility and the Lyapunov dimension, both for the time-correlated and randomized flows. The result is summarized in Fig. 5 where for comparison we plot the relation (3) for the $\delta$ correlated Kraichnan case. 


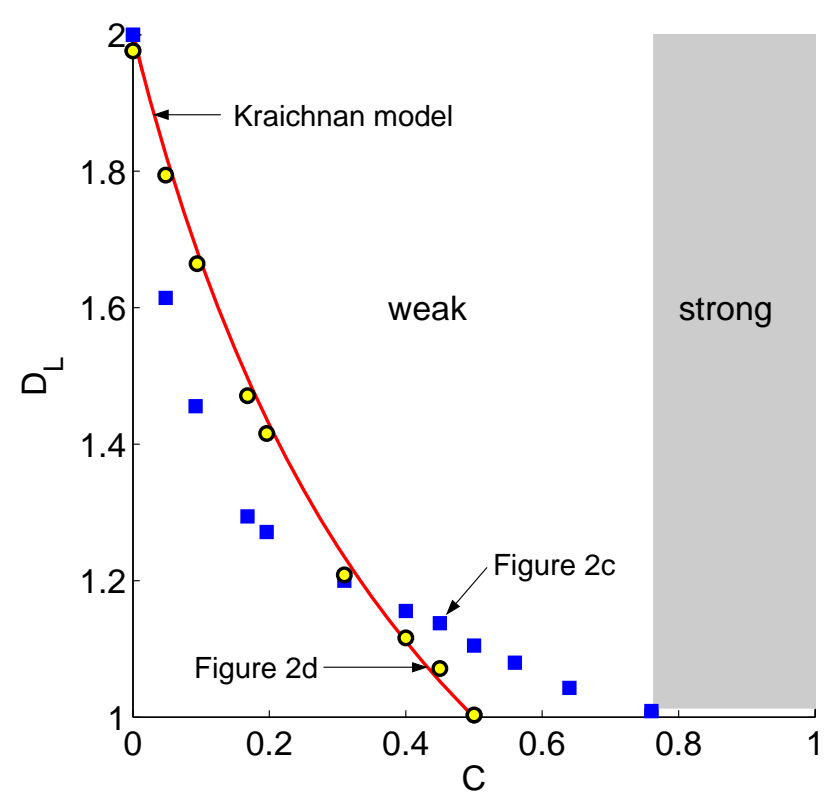

FIG. 5: Lyapunov dimension $D_{L}$ computed for different values of the compressibility $\mathcal{C}$ in accordance with (4). Data points for time-correlated flow are indicated by squares. The open circles are for the de-correlated flow. The line indicates the behavior for the compressible Kraichnan flow (cf. (3)). The gray shading in the background illustrates the border between weak and strong compressible regime for the timecorrelated flow. We have also indicated the data points in the plane that correspond with the tracer distributions as given in Fig. 2.

It is remarkable that the qualitative effect of time correlations on tracers distribution depends on the level of compressibility. For small values $\mathcal{C} \leq 0.3$, time correlations enhance the effects of compressibility, i.e. $D_{L}$ smaller than the predicted value by (3). The situation for larger values of $\mathcal{C}$ is more surprising as the Lyapunov dimension in correlated flow is larger than the value given by (3), i.e. the effects of compressibility are depleted with respect to the $\delta$-correlated case. The later situation is contrary to intuition according to which finite time correlations amplify the effects of compressibility. Consequently the transition point to the strong compressible regime, where $\lambda_{1} \leq 0$, moves up to the $\mathcal{C} \simeq 0.75$ larger than the prediction of (3) (cf. gray region in Fig. 5). This is again the point where the ratio $\frac{\lambda_{1}}{\lambda_{2}}$ gets zero for the correlated flow.

In summary, we have studied the effects of time correlations in the clustering of Lagrangian tracers advected on a free surface flow by direct numerical simulation of NS equations. Appealing to a numerical decomposition method we generate $2 d$ flows with variable degrees of compressibilities. The Lyapunov dimension decreases with the compressibility degree. Our simulations indicate that the effect of time correlation can go both ways. At low values of compressibility the Lyapunov dimension of the tracer distribution is smaller than the dimension in the time de-correlated flows. Yet as the compressibility gets higher this regime crosses over to a more interesting one where the time correlations increase the Lyapunov dimension. The point of phase transition to strong compressible regime is at a higher value, $\mathcal{C} \simeq 0.75$. The present paper is a first attempt to relate aspects of the solvable compressible Kraichnan model to the turbulent Navier-Stokes compressible flows. This suggests further investigations on the interrelated roles of the time correlations and the compressibility on the dispersion properties.

This work was supported by the Deutsche Forschungsgemeinschaft. The computations were carried out at the John von Neumann Institute for Computing at the Forschungszentrum Jülich and we are grateful for support. G. B. acknowledges hospitality of the Philipps Universität Marburg.

[1] J. Sommerer and E. Ott, Science 259, 335 (1993).

[2] S. Sundaram and L. R. Collins, J. Fluid Mech. 335, 75 (1997).

[3] V. I. Klyatskin and A. I. Saichev, JETP 84, 716 (1997).

[4] E. Balkovsky, G. Falkovich and A. Fouxon, Phys. Rev. Lett. 86, 2790 (2001).

[5] I. J. Benczik, Z. Toroczkai, and T. Tél, Phys. Rev. Lett. 89, 164501 (2002).

[6] T. Elperin, N. Kleeorin, V. S. L'vov, I. Rogachevskii, and D. Sokoloff, Phys. Rev. E 66, 036302 (2002).

[7] M. Wilkinson and B. Mehlig, Phys. Rev. E 68, 040101 (2003).

[8] J. Bec, Phys. Fluids 15, L81 (2003).

[9] I. M. Mazzitelli, D. Lohse, and F. Toschi, Phys. Fluids 15, L5 (2003).

[10] R. A. Shaw, Annu. Rev. Fluid Mech. 35, 183 (2003).

[11] J. Magnaudet and I. Eames, Annu. Rev. Fluid Mech. 32, 659 (2000).

[12] G. B. Deane and M. D. Stokes, Nature 418, 839 (2002).

[13] G. Falkovich, K. Gawędzki, and M. Vergassola, Rev. Mod. Phys. 73, 913 (2001).

[14] E. Ott, Chaos in Dynamical Systems, Cambridge University Press (1993).

[15] S. S. Girimaji and S. B. Pope, J. Fluid. Mech. 220, 427 (1990).

[16] M. Chaves, K. Gawędzki, P. Horvai, A. Kupiainen, and M. Vergassola, J. Stat. Phys. 113, 643 (2003).

[17] W. I. Goldburg, J. R. Cressman, Z. Vörös, B. Eckhardt and J. Schumacher, Phys. Rev. E 63, 065303 (R) (2001); B. Eckhardt and J. Schumacher, Phys. Rev. E 64, 016314 (2001).

[18] J. R. Cressman and W. I. Goldburg, J. Stat. Phys. 113, 875 (2003); J. R. Cressman, W. I. Goldburg, and J. Schumacher, Europhys. Lett. 66, 219 (2004).

[19] J. Schumacher and B. Eckhardt, Phys. Rev. E 66, 017303 (2002).

[20] J. R. Cressman, J. Davoudi, W. I. Goldburg, and J. Schumacher, New J. Phys. 6, 53 (2004). 